UMTG-266

\title{
Yangian symmetry and bound states in AdS/CFT boundary scattering
}

\author{
Changrim Ahn 1 and Rafael I. Nepomechie 2
}

\begin{abstract}
We consider the problem of boundary scattering for $Y=0$ maximal giant graviton branes. We show that the boundary $S$-matrix for the fundamental excitations has a Yangian symmetry. We then exploit this symmetry to determine the boundary $S$ matrix for two-particle bound states. We verify that this boundary $S$-matrix satisfies the boundary Yang-Baxter equations.
\end{abstract}

\footnotetext{
${ }^{1}$ Department of Physics, Ewha Womans University, Seoul 120-750, South Korea; ahn@ewha.ac.kr

2 Physics Department, P.O. Box 248046, University of Miami, Coral Gables, FL 33124 USA; nepomechie@physics.miami.edu
} 


\section{Introduction}

The AdS/CFT bulk $S$-matrix [1]-[3] has a remarkable Yangian symmetry $Y(s u(2 \mid 2))$ [4] 1 ] Since this $S$-matrix (which is for the fundamental excitations) can already be determined 2 from just the (centrally extended) su(2|2) symmetry, the further Yangian symmetry may appear to be only a mathematical curiosity. However, in order to construct higher dimensional ("bound state") $S$-matrices, the $s u(2 \mid 2)$ symmetry does not suffice [9]; and the Yangian symmetry can be used to completely determine the two-particle [9, 10] and general $l$-particle bound state bulk $S$-matrices [11. It is fortunate that such a general way of generating higherdimensional $S$-matrices has been found, since the fusion procedure [12] (which has played a very important role for conventional $S$-matrices) does not seem to work for AdS/CFT $S$-matrices. Knowledge of higher-dimensional $S$-matrices is necessary for deriving functional relations for the corresponding transfer matrices, thermodynamic Bethe ansatz equations, etc.

We initiate in this note an investigation of higher-dimensional AdS/CFT boundary Smatrices. For simplicity, we restrict to the case of open strings attached to so-called $Y=0$ maximal giant graviton branes [13]. The fundamental boundary $S$-matrix can be determined from $s u(1 \mid 2)$ symmetry [13, 14]. However, similarly to the bulk case, we find that this symmetry does not suffice to determine the bound state boundary $S$-matrices. We show that the fundamental boundary $S$-matrix has a residual Yangian symmetry. We then exploit this Yangian symmetry to determine the two-particle bound state boundary $S$-matrix. In contrast to the fundamental case, this boundary $S$-matrix is not diagonal. We verify that it satisfies the boundary Yang-Baxter equations. We formulate the discussion in terms of Zamolodchikov-Faddeev (ZF) operators [15, 16, which provide a convenient way of handling the intricate coproducts of the Yangian generators.

The outline of this paper is as follows. In Section 2 we consider the case of the fundamental excitations. After briefly reviewing the $s u(2 \mid 2)$ and Yangian symmetries of the bulk $S$-matrix, we show that the boundary $S$-matrix also has Yangian symmetry. In Section 3 we consider the case of two-particle bound states. After another brief review of the bulk symmetries in this higher-dimensional representation, we use the $s u(1 \mid 2)$ and Yangian symmetries to determine the boundary $S$-matrix. We conclude with a brief discussion of our results.

\footnotetext{
${ }^{1}$ For earlier investigations of Yangian symmetry in AdS/CFT, see for example [5]- 8 .

${ }^{2}$ We do not concern ourselves here with overall scalar factors. Also, we focus on just one copy of the $s u(2 \mid 2) S$-matrix; the full AdS/CFT $S$-matrix is a tensor product of two such copies.
} 


\section{Fundamental representation}

This section is devoted to the scattering of the fundamental excitations. We begin with a review of bulk scattering, and then turn to boundary scattering.

\section{$2.1 \quad$ Bulk scattering}

It is convenient to work with $\mathrm{ZF}$ operators. Following [3], we denote the fundamental ZF operators by $A_{i}^{\dagger}(p), i=1,2,3,4$. The operators with $i=1,2$ are bosonic, while the operators with $i=3,4$ are fermionic. These operators create asymptotic particle states of momentum $p$ when acting on the vacuum state $|0\rangle$. The matrix elements of the fundamental bulk $S$-matrix $S^{A A}\left(p_{1}, p_{2}\right)$ are defined by the relation

$$
A_{i}^{\dagger}\left(p_{1}\right) A_{j}^{\dagger}\left(p_{2}\right)=S_{i j}^{A A} \underset{i^{\prime} j^{\prime}}{ }\left(p_{1}, p_{2}\right) A_{j^{\prime}}^{\dagger}\left(p_{2}\right) A_{i^{\prime}}^{\dagger}\left(p_{1}\right)
$$

where summation over repeated indices is always understood.

The centrally extended $s u(2 \mid 2)$ algebra consists of the rotation generators $\mathbb{L}_{a}^{b}, \mathbb{R}_{\alpha}^{\beta}$, the supersymmetry generators $\mathbb{Q}_{\alpha}^{a}, \mathbb{Q}_{a}^{\dagger \alpha}$, and the central elements $\mathbb{C}, \mathbb{C}^{\dagger}, \mathbb{H}$. Latin indices $a, b, \ldots$ take values $\{1,2\}$, while Greek indices $\alpha, \beta, \ldots$ take values $\{3,4\}$. These generators have the following nontrivial commutation relations [2, 3]

$$
\begin{aligned}
{\left[\mathbb{L}_{a}^{b}, \mathbb{J}_{c}\right] } & =\delta_{c}^{b} \mathbb{J}_{a}-\frac{1}{2} \delta_{a}^{b} \mathbb{J}_{c}, \quad\left[\mathbb{R}_{\alpha}^{\beta}, \mathbb{J}_{\gamma}\right]=\delta_{\gamma}^{\beta} \mathbb{J}_{\alpha}-\frac{1}{2} \delta_{\alpha}^{\beta} \mathbb{J}_{\gamma}, \\
{\left[\mathbb{L}_{a}^{b}, \mathbb{J}^{c}\right] } & =-\delta_{a}^{c} \mathbb{J}^{b}+\frac{1}{2} \delta_{a}^{b} \mathbb{J}^{c}, \quad\left[\mathbb{R}_{\alpha}^{\beta}, \mathbb{J}^{\gamma}\right]=-\delta_{\alpha}^{\gamma} \mathbb{J}^{\beta}+\frac{1}{2} \delta_{\alpha}^{\beta} \mathbb{J}^{\gamma}, \\
\left\{\mathbb{Q}_{\alpha}^{a}, \mathbb{Q}_{\beta}^{b}\right\} & =\epsilon_{\alpha \beta} \epsilon^{a b} \mathbb{C}, \quad\left\{\mathbb{Q}_{a}^{\dagger \alpha}, \mathbb{Q}_{b}^{\dagger \beta}\right\}=\epsilon^{\alpha \beta} \epsilon_{a b} \mathbb{C}^{\dagger}, \\
\left\{\mathbb{Q}_{\alpha}^{a}, \mathbb{Q}_{b}^{\dagger \beta}\right\} & =\delta_{b}^{a} \mathbb{R}_{\alpha}^{\beta}+\delta_{\alpha}^{\beta} \mathbb{L}_{b}^{a}+\frac{1}{2} \delta_{b}^{a} \delta_{\alpha}^{\beta} \mathbb{H},
\end{aligned}
$$

where $\mathbb{J}_{i}\left(\mathbb{J}^{i}\right)$ denotes any lower (upper) index of a generator, respectively.

The action of the bosonic generators $\mathbb{L}_{a}{ }^{b}, \mathbb{R}_{\alpha}{ }^{\beta}$ on the ZF operators is given by

$$
\begin{aligned}
& \mathbb{L}_{a}^{b} A_{c}^{\dagger}(p)=\left(\delta_{c}^{b} \delta_{a}^{d}-\frac{1}{2} \delta_{a}^{b} \delta_{c}^{d}\right) A_{d}^{\dagger}(p)+A_{c}^{\dagger}(p) \mathbb{L}_{a}^{b}, \quad \mathbb{L}_{a}^{b} A_{\gamma}^{\dagger}(p)=A_{\gamma}^{\dagger}(p) \mathbb{L}_{a}^{b}, \\
& \mathbb{R}_{\alpha}^{\beta} A_{\gamma}^{\dagger}(p)=\left(\delta_{\gamma}^{\beta} \delta_{\alpha}^{\delta}-\frac{1}{2} \delta_{\alpha}^{\beta} \delta_{\gamma}^{\delta}\right) A_{\delta}^{\dagger}(p)+A_{\gamma}^{\dagger}(p) \mathbb{R}_{\alpha}{ }^{\beta}, \quad \mathbb{R}_{\alpha}{ }^{\beta} A_{c}^{\dagger}(p)=A_{c}^{\dagger}(p) \mathbb{R}_{\alpha}{ }^{\beta}
\end{aligned}
$$

Moreover, the action of the supersymmetry generators on the ZF operators is given by (see 
Eq. (4.21) in [3] and [14])

$$
\begin{aligned}
\mathbb{Q}_{\alpha}{ }^{a} A_{b}^{\dagger}(p) & =e^{-i p / 2}\left[a(p) \delta_{b}^{a} A_{\alpha}^{\dagger}(p)+A_{b}^{\dagger}(p) \mathbb{Q}_{\alpha}^{a}\right] \\
\mathbb{Q}_{\alpha}{ }^{a} A_{\beta}^{\dagger}(p) & =e^{-i p / 2}\left[b(p) \epsilon_{\alpha \beta} \epsilon^{a b} A_{b}^{\dagger}(p)-A_{\beta}^{\dagger}(p) \mathbb{Q}_{\alpha}^{a}\right] \\
\mathbb{Q}_{a}^{\dagger \alpha} A_{b}^{\dagger}(p) & =e^{i p / 2}\left[c(p) \epsilon_{a b} \epsilon^{\alpha \beta} A_{\beta}^{\dagger}(p)+A_{b}^{\dagger}(p) \mathbb{Q}_{a}^{\dagger \alpha}\right] \\
\mathbb{Q}_{a}^{\dagger \alpha} A_{\beta}^{\dagger}(p) & =e^{i p / 2}\left[d(p) \delta_{\beta}^{\alpha} A_{a}^{\dagger}(p)-A_{\beta}^{\dagger}(p) \mathbb{Q}_{a}^{\dagger \alpha}\right]
\end{aligned}
$$

It follows that the action of the central charges on the ZF operators is given by

$$
\begin{aligned}
\mathbb{C} A_{i}^{\dagger}(p) & =e^{-i p}\left[a(p) b(p) A_{i}^{\dagger}(p)+A_{i}^{\dagger}(p) \mathbb{C}\right], \\
\mathbb{C}^{\dagger} A_{i}^{\dagger}(p) & =e^{i p}\left[c(p) d(p) A_{i}^{\dagger}(p)+A_{i}^{\dagger}(p) \mathbb{C}^{\dagger}\right], \\
\mathbb{H} A_{i}^{\dagger}(p) & =[a(p) d(p)+b(p) c(p)] A_{i}^{\dagger}(p)+A_{i}^{\dagger}(p) \mathbb{H} .
\end{aligned}
$$

Arutyunov-Frolov-Zamaklar work with a different set of relations for the supersymmetry generators which involve the world-sheet momentum operator (see Eq. (4.15) in [3]). However, as noted in [14], the relations (2.4) are more natural when dealing with a boundary.

The ZF operators form a representation of the symmetry algebra provided $a d-b c=1$. The parameters can be chosen as follows [2, 9]

$$
a=\sqrt{\frac{g}{2 l}} \eta, \quad b=\sqrt{\frac{g}{2 l}} \frac{i}{\eta}\left(\frac{x^{+}}{x^{-}}-1\right), \quad c=-\sqrt{\frac{g}{2 l}} \frac{\eta}{x^{+}}, \quad d=\sqrt{\frac{g}{2 l}} \frac{x^{+}}{i \eta}\left(1-\frac{x^{-}}{x^{+}}\right),
$$

where

$$
x^{+}+\frac{1}{x^{+}}-x^{-}-\frac{1}{x^{-}}=\frac{2 l i}{g}, \quad \frac{x^{+}}{x^{-}}=e^{i p},
$$

and $l=1$ for the fundamental case under consideration in this section. Moreover, following [9], we take

$$
\eta=e^{i p / 4} \sqrt{i\left(x^{-}-x^{+}\right)}
$$

which has an extra factor $e^{i p / 4}$ compared with the corresponding quantity in [3, 14].

The fundamental bulk $S$-matrix (2.1) is determined by this $s u(2 \mid 2)$ symmetry [2, 3]. For 
convenience, we reproduce here the result for the nonzero matrix elements: 3

$$
\begin{aligned}
S_{a a}^{a a} & =\mathcal{A}, \quad S_{\alpha \alpha}^{\alpha \alpha}=\mathcal{D}, \\
S_{a b}^{a b} & =\frac{1}{2}(\mathcal{A}-\mathcal{B}), \quad S_{a b}^{b a}=\frac{1}{2}(\mathcal{A}+\mathcal{B}), \\
S_{\alpha \beta}^{\alpha \beta} & =\frac{1}{2}(\mathcal{D}-\mathcal{E}), \quad S_{\alpha \beta}^{\beta \alpha}=\frac{1}{2}(\mathcal{D}+\mathcal{E}), \\
S_{a b}^{\alpha \beta} & =-\frac{1}{2} \epsilon_{a b} \epsilon^{\alpha \beta} \mathcal{C}, \quad S_{\alpha \beta}^{a b}=-\frac{1}{2} \epsilon^{a b} \epsilon_{\alpha \beta} \mathcal{F}, \\
S_{a \alpha}^{a \alpha} & =\mathcal{G}, \quad S_{a \alpha}^{\alpha a}=\mathcal{H}, \quad S_{\alpha a}^{a \alpha}=\mathcal{K}, \quad S_{\alpha a}^{\alpha a}=\mathcal{L},
\end{aligned}
$$

where $a, b \in\{1,2\}$ with $a \neq b ; \alpha, \beta \in\{3,4\}$ with $\alpha \neq \beta$; and

$$
\begin{aligned}
\mathcal{A} & =S_{0} \frac{x_{2}^{-}-x_{1}^{+}}{x_{2}^{+}-x_{1}^{-}} \frac{\eta_{1} \eta_{2}}{\tilde{\eta}_{1} \tilde{\eta}_{2}}, \\
\mathcal{B} & =-S_{0}\left[\frac{x_{2}^{-}-x_{1}^{+}}{x_{2}^{+}-x_{1}^{-}}+2 \frac{\left(x_{1}^{-}-x_{1}^{+}\right)\left(x_{2}^{-}-x_{2}^{+}\right)\left(x_{2}^{-}+x_{1}^{+}\right)}{\left(x_{1}^{-}-x_{2}^{+}\right)\left(x_{1}^{-} x_{2}^{-}-x_{1}^{+} x_{2}^{+}\right)}\right] \frac{\eta_{1} \eta_{2}}{\tilde{\eta}_{1} \tilde{\eta}_{2}} \\
\mathcal{C} & =S_{0} \frac{2 i x_{1}^{-} x_{2}^{-}\left(x_{1}^{+}-x_{2}^{+}\right) \eta_{1} \eta_{2}}{x_{1}^{+} x_{2}^{+}\left(x_{1}^{-}-x_{2}^{+}\right)\left(1-x_{1}^{-} x_{2}^{-}\right)}, \quad \mathcal{D}=-S_{0}, \\
\mathcal{E} & =S_{0}\left[1-2 \frac{\left(x_{1}^{-}-x_{1}^{+}\right)\left(x_{2}^{-}-x_{2}^{+}\right)\left(x_{1}^{-}+x_{2}^{+}\right)}{\left(x_{1}^{-}-x_{2}^{+}\right)\left(x_{1}^{-} x_{2}^{-}-x_{1}^{+} x_{2}^{+}\right)}\right] \\
\mathcal{F} & =S_{0} \frac{2 i\left(x_{1}^{-}-x_{1}^{+}\right)\left(x_{2}^{-}-x_{2}^{+}\right)\left(x_{1}^{+}-x_{2}^{+}\right)}{\left(x_{1}^{-}-x_{2}^{+}\right)\left(1-x_{1}^{-} x_{2}^{-}\right) \tilde{\eta}_{1} \tilde{\eta}_{2}}, \\
\mathcal{G} & =S_{0} \frac{\left(x_{2}^{-}-x_{1}^{-}\right)}{\left(x_{2}^{+}-x_{1}^{-}\right)} \frac{\eta_{1}}{\tilde{\eta}_{1}}, \quad \mathcal{H}=S_{0} \frac{\left(x_{2}^{+}-x_{2}^{-}\right)}{\left(x_{1}^{-}-x_{2}^{+}\right)} \frac{\eta_{1}}{\tilde{\eta}_{2}} \\
\mathcal{K} & =S_{0} \frac{\left(x_{1}^{+}-x_{1}^{-}\right)}{\left(x_{1}^{-}-x_{2}^{+}\right)} \frac{\eta_{2}}{\tilde{\eta}_{1}}, \quad \mathcal{L}=S_{0} \frac{\left(x_{1}^{+}-x_{2}^{+}\right)}{\left(x_{1}^{-}-x_{2}^{+}\right)} \frac{\eta_{2}}{\tilde{\eta}_{2}}
\end{aligned}
$$

where

$$
x_{i}^{ \pm}=x^{ \pm}\left(p_{i}\right), \quad \eta_{1}=\eta\left(p_{1}\right) e^{i p_{2} / 2}, \quad \eta_{2}=\eta\left(p_{2}\right), \quad \tilde{\eta}_{1}=\eta\left(p_{1}\right), \quad \tilde{\eta}_{2}=\eta\left(p_{2}\right) e^{i p_{1} / 2},
$$

and $\eta(p)$ is given in (2.8). This $S$-matrix satisfies the standard Yang-Baxter equation.

Following [4, 11], for each $s u(2 \mid 2)$ generator $\mathbb{J}$, we denote the corresponding Yangian $Y(s u(2 \mid 2))$ generator (in the evaluation representation) by

$$
\hat{\mathbb{J}}=-\frac{1}{2} i g u \mathbb{J},
$$

\footnotetext{
${ }^{3}$ In order to streamline the notation, here we denote the $S$-matrix element $S_{i j}^{A A} i_{i j}^{j^{\prime}}\left(p_{1}, p_{2}\right)$ simply by $S_{i j}^{i^{\prime} j^{\prime}}$; i.e., we drop both the $A A$ label and the arguments $\left(p_{1}, p_{2}\right)$.
} 
where

$$
u=\frac{1}{2}\left(x^{+}+\frac{1}{x^{+}}+x^{-}+\frac{1}{x^{-}}\right) .
$$

The action of the Yangian generators on the ZF operators can be inferred from the coproducts given in [4, 11] and the relations (2.3)-(2.5). For example, from the coproduct for $\hat{\mathbb{L}}_{2}^{1}$

$$
\begin{aligned}
\Delta\left(\hat{\mathbb{L}}_{2}{ }^{1}\right)= & \hat{\mathbb{L}}_{2}{ }^{1} \otimes \mathbb{I}+\mathbb{I} \otimes \hat{\mathbb{L}}_{2}{ }^{1}+\frac{1}{2} \mathbb{L}_{2}{ }^{c} \otimes \mathbb{L}_{c}{ }^{1}-\frac{1}{2} \mathbb{L}_{c}{ }^{1} \otimes \mathbb{L}_{2}{ }^{c} \\
& -\frac{1}{2} \mathbb{Q}_{2}^{\dagger \gamma} \otimes \mathbb{Q}_{\gamma}{ }^{1}-\frac{1}{2} \mathbb{Q}_{\gamma}{ }^{1} \otimes \mathbb{Q}_{2}^{\dagger \gamma},
\end{aligned}
$$

we obtain the relations (which we shall use later)

$$
\begin{aligned}
\hat{\mathbb{L}}_{2}{ }^{1} A_{1}^{\dagger}(p) & =-\frac{1}{2} i g u A_{2}^{\dagger}(p)+A_{1}^{\dagger}(p) \hat{\mathbb{L}}_{2}{ }^{1}-\frac{1}{2} A_{1}^{\dagger}(p) \mathbb{L}_{2}{ }^{1}+\frac{1}{2} A_{2}^{\dagger}(p)\left(\mathbb{L}_{1}{ }^{1}-\mathbb{L}_{2}{ }^{2}\right) \\
& +\frac{1}{2} c(p) A_{4}^{\dagger}(p) \mathbb{Q}_{3}{ }^{1}-\frac{1}{2} c(p) A_{3}^{\dagger}(p) \mathbb{Q}_{4}{ }^{1}-\frac{1}{2} a(p) A_{3}^{\dagger}(p) \mathbb{Q}_{2}^{\dagger 3}-\frac{1}{2} a(p) A_{4}^{\dagger}(p) \mathbb{Q}_{2}^{\dagger 4} \\
\hat{\mathbb{L}}_{2}{ }^{1} A_{2}^{\dagger}(p) & =A_{2}^{\dagger}(p) \hat{\mathbb{L}}_{2}{ }^{1}+\frac{1}{2} A_{2}^{\dagger}(p) \mathbb{L}_{2}{ }^{1} \\
\hat{\mathbb{L}}_{2}{ }^{1} A_{3}^{\dagger}(p) & =A_{3}^{\dagger}(p) \hat{\mathbb{L}}_{2}{ }^{1}+\frac{1}{2} d(p) A_{2}^{\dagger}(p) \mathbb{Q}_{3}{ }^{1}-\frac{1}{2} b(p) A_{2}^{\dagger}(p) \mathbb{Q}_{2}^{\dagger 4} \\
\hat{\mathbb{L}}_{2}{ }^{1} A_{4}^{\dagger}(p) & =A_{4}^{\dagger}(p) \hat{\mathbb{L}}_{2}{ }^{1}+\frac{1}{2} d(p) A_{2}^{\dagger}(p) \mathbb{Q}_{4}{ }^{1}+\frac{1}{2} b(p) A_{2}^{\dagger}(p) \mathbb{Q}_{2}^{\dagger 3} .
\end{aligned}
$$

We have verified that these relations, together with many others which we have not listed here, are consistent with the bulk $S$-matrix (2.9), (2.10), thereby confirming the $Y(s u(2 \mid 2))$ Yangian symmetry of the latter.

\subsection{Boundary scattering}

We consider now the problem of boundary scattering for the fundamental excitations of open strings attached to $Y=0$ maximal giant graviton branes [13. In order to describe boundary scattering, we extend (following [14]) the bulk ZF algebra (2.1) by introducing a boundary operator $\mathbf{B}$ which creates the boundary-theory vacuum state $|0\rangle_{B}=\mathbf{B}|0\rangle$ [17]. Since there is no boundary degree of freedom for the $Y=0$ brane, the boundary operator is a scalar. We define the fundamental (right) boundary $S$-matrix $R^{A}(p)$ by 4

$$
A_{i}^{\dagger}(p) \mathbf{B}=R_{i}^{A i^{\prime}}(p) A_{i^{\prime}}^{\dagger}(-p) \mathbf{B} .
$$

\footnotetext{
${ }^{4}$ We consider here just a right boundary, since a left boundary can be treated in a similar way [14].
} 
As for the bulk, the boundary $S$-matrix can be determined from the symmetry of the problem. Indeed, the $Y=0$ brane preserves only an $s u(1 \mid 2)$ subalgebra [13], which consists of the generators

$$
\mathbb{L}_{1}{ }^{1}, \quad \mathbb{L}_{2}{ }^{2}, \quad \mathbb{H}, \quad \mathbb{R}_{\alpha}^{\beta}, \quad \mathbb{Q}_{\alpha}{ }^{1}, \quad \mathbb{Q}_{1}^{\dagger \alpha} \quad \text { with } \alpha, \beta \in\{3,4\} .
$$

The vacuum state $|0\rangle_{B}$ is annihilated by each of these generators. Consider now one-particle states $A_{i}^{\dagger}(p)|0\rangle_{B}$. Invariance under $\mathbb{L}_{1}{ }^{1}$ and $\mathbb{R}_{\alpha}{ }^{\beta}$ implies that the boundary $S$-matrix is diagonal, with the structure

$$
R^{A}(p)=\operatorname{diag}\left(r_{1}, r_{2}, r, r\right) .
$$

Invariance under $\mathbb{Q}_{3}{ }^{1}$ then determines the diagonal matrix elements,

$$
\begin{aligned}
& \frac{r_{1}}{r}=e^{-i p} \frac{a(p)}{a(-p)}=e^{-i p / 2}, \\
& \frac{r_{2}}{r}=e^{i p} \frac{b(-p)}{b(p)}=-e^{i p / 2} .
\end{aligned}
$$

This result differs from the one in [14] due to the different expression (2.8) for $\eta(p)$. This matrix satisfies the standard boundary Yang-Baxter equation

$$
\begin{aligned}
& S_{12}^{A A}\left(p_{1}, p_{2}\right) R_{1}^{A}\left(p_{1}\right) S_{21}^{A A}\left(p_{2},-p_{1}\right) R_{2}^{A}\left(p_{2}\right) \\
& \quad=R_{2}^{A}\left(p_{2}\right) S_{12}^{A A}\left(p_{1},-p_{2}\right) R_{1}^{A}\left(p_{1}\right) S_{21}^{A A}\left(-p_{2},-p_{1}\right),
\end{aligned}
$$

where

$$
S_{21}^{A A}\left(p_{1}, p_{2}\right)=\mathcal{P}_{12} S_{12}^{A A}\left(p_{1}, p_{2}\right) \mathcal{P}_{12}=S_{12}^{A A}\left(p_{2}, p_{1}\right)^{-1},
$$

and $\mathcal{P}$ is the permutation matrix.

We now show that this boundary $S$-matrix also has a residual Yangian symmetry. Indeed, consider the charge $\tilde{\mathbb{Q}}$ defined by

$$
\tilde{\mathbb{Q}}=\hat{\mathbb{L}}_{2}{ }^{1}+\frac{1}{2}\left(\mathbb{L}_{2}{ }^{1} \mathbb{L}_{1}^{1}-\mathbb{L}_{2}{ }^{1} \mathbb{L}_{2}{ }^{2}-\mathbb{Q}_{2}^{\dagger 3} \mathbb{Q}_{3}{ }^{1}-\mathbb{Q}_{2}^{\dagger 4} \mathbb{Q}_{4}{ }^{1}\right),
$$

which we shall assume is also conserved. This charge has the following action on the ZF operators,

$$
\begin{aligned}
\tilde{\mathbb{Q}} A_{1}^{\dagger}(p)= & {\left[-\frac{1}{2} i g u+\frac{1}{2}-a(p) d(p)\right] A_{2}^{\dagger}(p)+A_{2}^{\dagger}(p)\left(\mathbb{L}_{1}{ }^{1}-\mathbb{L}_{2}{ }^{2}\right) } \\
& +c(p) A_{4}^{\dagger}(p) \mathbb{Q}_{3}{ }^{1}-c(p) A_{3}^{\dagger}(p) \mathbb{Q}_{4}{ }^{1}+A_{1}^{\dagger}(p) \tilde{\mathbb{Q}}, \\
\tilde{\mathbb{Q}} A_{2}^{\dagger}(p)= & A_{2}^{\dagger}(p) \tilde{\mathbb{Q}}, \\
\tilde{\mathbb{Q}} A_{3}^{\dagger}(p)= & d(p) A_{2}^{\dagger}(p) \mathbb{Q}_{3}{ }^{1}+A_{3}^{\dagger}(p) \tilde{\mathbb{Q}}, \\
\tilde{\mathbb{Q}} A_{4}^{\dagger}(p)= & d(p) A_{2}^{\dagger}(p) \mathbb{Q}_{4}{ }^{1}+A_{4}^{\dagger}(p) \tilde{\mathbb{Q}},
\end{aligned}
$$


as can be verified with the help of (2.15). The key point is that all the symmetry generators appearing on the RHS of (2.23) annihilate the vacuum state. A similar construction has appeared in e.g. [18, 19].

In order to see the symmetry of the boundary $S$-matrix, consider the action of $\tilde{\mathbb{Q}}$ on the one-particle state $A_{1}^{\dagger}(p)|0\rangle_{B}$. Using (2.23), we obtain

$$
\tilde{\mathbb{Q}} A_{1}^{\dagger}(p)|0\rangle_{B}=f(p) A_{2}^{\dagger}(p)|0\rangle_{B}=r_{2} f(p) A_{2}^{\dagger}(-p)|0\rangle_{B}
$$

where in the first equality we have also introduced

$$
f(p)=-\frac{1}{2} i g u+\frac{1}{2}-a(p) d(p)=-\frac{1}{2}[i g u+a(p) d(p)+b(p) c(p)],
$$

and we have used the fact that $\tilde{\mathbb{Q}}$ and the $s u(1 \mid 2)$ generators (2.17) annihilate the vacuum state; and in the second equality we have "reflected" using (2.16), (2.18) . Reversing the order of operations, we obtain

$$
\tilde{\mathbb{Q}} A_{1}^{\dagger}(p)|0\rangle_{B}=r_{1} \tilde{\mathbb{Q}} A_{1}^{\dagger}(-p)|0\rangle_{B}=r_{1} f(-p) A_{2}^{\dagger}(-p)|0\rangle_{B}
$$

Comparing (2.24) and (2.26), we arrive at the relation

$$
\frac{r_{1}}{r_{2}}=\frac{f(p)}{f(-p)}=-e^{-i p}
$$

which is consistent with the result (2.19). We conclude that the charge $\tilde{\mathbb{Q}}(2.22)$ constructed with the Yangian generator $\hat{\mathbb{L}}_{2}^{1}$ is a symmetry of the boundary $S$-matrix. Additional charges of this sort can be constructed, but we shall not need them here.

\section{Two-particle bound state representation}

We now proceed to determine the boundary $S$-matrix for two-particle bound states. We begin by reviewing some necessary results about such states and their bulk scattering.

\subsection{Bulk scattering}

The two-particle bound states form an 8-dimensional (atypical totally symmetric) representation of $s u(2 \mid 2)$. Following the convenient superspace formalism in [9], the $s u(2 \mid 2)$ generators can be represented by differential operators on a vector space of analytic functions of two 
bosonic variables $w_{a}$ and two fermionic variables $\theta_{\alpha}$, as follows:

$$
\begin{array}{rlrl}
\mathbb{L}_{a}^{b} & =w_{a} \frac{\partial}{\partial w_{b}}-\frac{1}{2} \delta_{a}^{b} w_{c} \frac{\partial}{\partial w_{c}}, & \mathbb{R}_{\alpha}^{\beta}=\theta_{\alpha} \frac{\partial}{\partial \theta_{\beta}}-\frac{1}{2} \delta_{\alpha}^{\beta} \theta_{\gamma} \frac{\partial}{\partial \theta_{\gamma}} \\
\mathbb{Q}_{\alpha}^{a} & =a \theta_{\alpha} \frac{\partial}{\partial w_{a}}+b \epsilon^{a b} \epsilon_{\alpha \beta} w_{b} \frac{\partial}{\partial \theta_{\beta}}, & \mathbb{Q}_{a}^{\dagger \alpha}=d w_{a} \frac{\partial}{\partial \theta_{\alpha}}+c \epsilon_{a b} \epsilon^{\alpha \beta} \theta_{\beta} \frac{\partial}{\partial w_{b}} \\
\mathbb{C} & =a b\left(w_{a} \frac{\partial}{\partial w_{a}}+\theta_{\alpha} \frac{\partial}{\partial \theta_{\alpha}}\right), & \mathbb{C}^{\dagger}=c d\left(w_{a} \frac{\partial}{\partial w_{a}}+\theta_{\alpha} \frac{\partial}{\partial \theta_{\alpha}}\right), \\
\mathbb{H} & =(a d+b c)\left(w_{a} \frac{\partial}{\partial w_{a}}+\theta_{\alpha} \frac{\partial}{\partial \theta_{\alpha}}\right) .
\end{array}
$$

The basis vectors $\left|e_{i}\right\rangle$ of the fundamental representation are $\left|e_{a}\right\rangle=w_{a},\left|e_{\alpha}\right\rangle=\theta_{\alpha}$; and the basis vectors $\left|e_{J}\right\rangle$ of the two-particle bound state representation are [9]

$$
\begin{array}{llll}
\left|e_{1}\right\rangle=\frac{w_{1} w_{1}}{\sqrt{2}}, & \left|e_{2}\right\rangle=w_{1} w_{2}, & \left|e_{3}\right\rangle=\frac{w_{2} w_{2}}{\sqrt{2}}, & \left|e_{4}\right\rangle=\theta_{3} \theta_{4}, \\
\left|e_{5}\right\rangle=w_{1} \theta_{3}, & \left|e_{6}\right\rangle=w_{1} \theta_{4}, & \left|e_{7}\right\rangle=w_{2} \theta_{3}, & \left|e_{8}\right\rangle=w_{2} \theta_{4} .
\end{array}
$$

We introduce corresponding ZF operators $B_{J}^{\dagger}(p), J=1, \ldots, 8$ for the two-particle bound states. The operators with $J=1, \ldots, 4$ are bosonic, while the operators with $J=5, \ldots, 8$ are fermionic. The bulk $S$-matrix $S^{A B}\left(p_{1}, p_{2}\right)$ is defined by

$$
A_{i}^{\dagger}\left(p_{1}\right) B_{J}^{\dagger}\left(p_{2}\right)=S_{i J}^{A B} i_{i J^{\prime}}^{J^{\prime}}\left(p_{1}, p_{2}\right) B_{J^{\prime}}^{\dagger}\left(p_{2}\right) A_{i^{\prime}}^{\dagger}\left(p_{1}\right)
$$

and the bulk $S$-matrix $S^{B B}\left(p_{1}, p_{2}\right)$ is defined by

$$
B_{I}^{\dagger}\left(p_{1}\right) B_{J}^{\dagger}\left(p_{2}\right)=S_{I J}^{B B I^{\prime} J^{\prime}}\left(p_{1}, p_{2}\right) B_{J^{\prime}}^{\dagger}\left(p_{2}\right) B_{I^{\prime}}^{\dagger}\left(p_{1}\right) .
$$

Both $S$-matrices are given in $[9]$. 5

The action of the bosonic $s u(2 \mid 2)$ generators $\mathbb{J} \in\left\{\mathbb{L}_{a}^{b}, \mathbb{R}_{\alpha}{ }^{\beta}\right\}$ on these ZF operators is given by (cf. (2.3) $)$

$$
\mathbb{J} B_{J}^{\dagger}(p)=(\mathbb{J})_{J}^{K} B_{K}^{\dagger}(p)+B_{J}^{\dagger}(p) \mathbb{J},
$$

and the action of the supersymmetry generators is given by (cf. (2.4)

$$
\begin{aligned}
\mathbb{Q}_{\alpha}^{a} B_{J}^{\dagger}(p) & =e^{-i p / 2}\left[\left(\mathbb{Q}_{\alpha}^{a}\right)_{J}^{K} B_{K}^{\dagger}(p)+(-1)^{\epsilon_{J}} B_{J}^{\dagger}(p) \mathbb{Q}_{\alpha}^{a}\right], \\
\mathbb{Q}_{a}^{\dagger \alpha} B_{J}^{\dagger}(p) & =e^{i p / 2}\left[\left(\mathbb{Q}_{a}^{\dagger \alpha}\right)_{J}^{K} B_{K}^{\dagger}(p)+(-1)^{\epsilon_{J}} B_{J}^{\dagger}(p) \mathbb{Q}_{a}^{\dagger \alpha}\right],
\end{aligned}
$$

\footnotetext{
${ }^{5}$ There are two typos in the coefficients of $S^{A B}$ listed in Section 6.1.2 of [9]. In $a_{13}$, the factor in the numerator $\left(x_{1}^{-}-y_{2}^{+}\right)$should be instead $\left(x_{1}^{+}-y_{2}^{+}\right)$; i.e., the $x_{1}^{-}$should be changed to $x_{1}^{+}$. And in $a_{14}$, the factor in the numerator $\left(1-y_{2}^{-} x_{1}^{-}\right)$should be instead $\left(1-y_{2}^{-} x_{1}^{+}\right)$; i.e., the $x_{1}^{-}$should be changed to $x_{1}^{+}$.
} 
where the matrix elements $(\mathbb{J})_{J}^{K},\left(\mathbb{Q}_{\alpha}^{a}\right)_{J}^{K},\left(\mathbb{Q}_{a}^{\dagger \alpha}\right)_{J}^{K}$ can be computed from (3.1), (3.2), and are provided for the reader's convenience in the Appendix; $\epsilon_{J}$ is the Grassmann parity,

$$
\epsilon_{J}=\left\{\begin{array}{ll}
0 & \text { for } J=1, \ldots, 4 \\
1 & \text { for } J=5, \ldots, 8
\end{array},\right.
$$

and $a, b, c, d, x^{ \pm}$are given by (2.6) , (2.7) with now $l=2$. Moreover, for the central charges,

$$
\begin{aligned}
\mathbb{C} B_{J}^{\dagger}(p) & =e^{-i p}\left[2 a(p) b(p) B_{J}^{\dagger}(p)+B_{J}^{\dagger}(p) \mathbb{C}\right], \\
\mathbb{C}^{\dagger} B_{J}^{\dagger}(p) & =e^{i p}\left[2 c(p) d(p) B_{J}^{\dagger}(p)+B_{J}^{\dagger}(p) \mathbb{C}^{\dagger}\right], \\
\mathbb{H} B_{J}^{\dagger}(p) & =2[a(p) d(p)+b(p) c(p)] B_{J}^{\dagger}(p)+B_{J}^{\dagger}(p) \mathbb{H} .
\end{aligned}
$$

The action of the $Y(s u(2 \mid 2))$ Yangian generators on the ZF operators can be inferred, similarly to the case of the fundamental representation, from the coproducts given in [4, 11] and the relations (3.5), (3.6). In particular, from the coproduct (2.14), we obtain

$$
\begin{aligned}
\hat{\mathbb{L}}_{2}{ }^{1} B_{1}^{\dagger}(p) & =-\frac{\sqrt{2}}{2} i g u B_{2}^{\dagger}(p)+B_{1}^{\dagger}(p) \hat{\mathbb{L}}_{2}{ }^{1}-B_{1}^{\dagger}(p) \mathbb{L}_{2}{ }^{1}+\frac{\sqrt{2}}{2} B_{2}^{\dagger}(p)\left(\mathbb{L}_{1}{ }^{1}-\mathbb{L}_{2}{ }^{2}\right) \\
& +\frac{\sqrt{2}}{2} c(p) B_{6}^{\dagger}(p) \mathbb{Q}_{3}{ }^{1}-\frac{\sqrt{2}}{2} c(p) B_{5}^{\dagger}(p) \mathbb{Q}_{4}{ }^{1}-\frac{\sqrt{2}}{2} a(p) B_{5}^{\dagger}(p) \mathbb{Q}_{2}^{\dagger 3}-\frac{\sqrt{2}}{2} a(p) B_{6}^{\dagger}(p) \mathbb{Q}_{2}^{\dagger 4}, \\
\hat{\mathbb{L}}_{2}{ }^{1} B_{2}^{\dagger}(p)= & -\frac{\sqrt{2}}{2} i g u B_{3}^{\dagger}(p)+B_{2}^{\dagger}(p) \hat{\mathbb{L}}_{2}{ }^{1}+\frac{\sqrt{2}}{2} B_{3}^{\dagger}(p)\left(\mathbb{L}_{1}{ }^{1}-\mathbb{L}_{2}{ }^{2}\right) \\
& +\frac{1}{2} c(p) B_{8}^{\dagger}(p) \mathbb{Q}_{3}{ }^{1}-\frac{1}{2} c(p) B_{7}^{\dagger}(p) \mathbb{Q}_{4}{ }^{1}-\frac{1}{2} a(p) B_{7}^{\dagger}(p) \mathbb{Q}_{2}^{\dagger 3}-\frac{1}{2} a(p) B_{8}^{\dagger}(p) \mathbb{Q}_{2}^{\dagger 4}, \\
\hat{\mathbb{L}}_{2}{ }^{1} B_{3}^{\dagger}(p)= & B_{3}^{\dagger}(p) \hat{\mathbb{L}}_{2}{ }^{1}+B_{3}^{\dagger}(p) \mathbb{L}_{2}{ }^{1}, \\
\hat{\mathbb{L}}_{2}{ }^{1} B_{4}^{\dagger}(p)= & B_{4}^{\dagger}(p) \hat{\mathbb{L}}_{2}{ }^{1}-\frac{1}{2} d(p) B_{8}^{\dagger}(p) \mathbb{Q}_{3}{ }^{1}+\frac{1}{2} d(p) B_{7}^{\dagger}(p) \mathbb{Q}_{4}{ }^{1}+\frac{1}{2} b(p) B_{7}^{\dagger}(p) \mathbb{Q}_{2}^{\dagger 3}+\frac{1}{2} b(p) B_{8}^{\dagger}(p) \mathbb{Q}_{2}^{\dagger 4}, \\
\hat{\mathbb{L}}_{2}{ }^{1} B_{5}^{\dagger}(p)= & -\frac{1}{2} i g u B_{7}^{\dagger}(p)+B_{5}^{\dagger}(p) \hat{\mathbb{L}}_{2}{ }^{1}-\frac{1}{2} B_{5}^{\dagger}(p) \mathbb{L}_{2}{ }^{1}+\frac{1}{2} B_{7}^{\dagger}(p)\left(\mathbb{L}_{1}{ }^{1}-\mathbb{L}_{2}{ }^{2}\right) \\
& +\frac{1}{2}\left[d(p) B_{2}^{\dagger}(p)+c(p) B_{4}^{\dagger}(p)\right] \mathbb{Q}_{3}{ }^{1}-\frac{1}{2}\left[a(p) B_{4}^{\dagger}(p)+b(p) B_{2}^{\dagger}(p)\right] \mathbb{Q}_{2}^{\dagger 4}, \\
\hat{\mathbb{L}}_{2}{ }^{1} B_{6}^{\dagger}(p)= & -\frac{1}{2} i g u B_{8}^{\dagger}(p)+B_{6}^{\dagger}(p) \hat{\mathbb{L}}_{2}{ }^{1}-\frac{1}{2} B_{6}^{\dagger}(p) \mathbb{L}_{2}{ }^{1}+\frac{1}{2} B_{8}^{\dagger}(p)\left(\mathbb{L}_{1}{ }^{1}-\mathbb{L}_{2}{ }^{2}\right) \\
& +\frac{1}{2}\left[d(p) B_{2}^{\dagger}(p)+c(p) B_{4}^{\dagger}(p)\right] \mathbb{Q}_{4}{ }^{1}+\frac{1}{2}\left[a(p) B_{4}^{\dagger}(p)+b(p) B_{2}^{\dagger}(p)\right] \mathbb{Q}_{2}^{\dagger 3}, \\
\hat{\mathbb{L}}_{2}{ }^{1} B_{8}^{\dagger}(p)= & B_{8}^{\dagger}(p) \hat{\mathbb{L}}_{2}{ }^{1}+\frac{1}{2} B_{8}^{\dagger}(p) \mathbb{L}_{2}{ }^{1}+\frac{\sqrt{2}}{2} d(p) B_{3}^{\dagger}(p) \mathbb{Q}_{4}{ }^{1}+\frac{\sqrt{2}}{2} b(p) B_{3}^{\dagger}(p) \mathbb{Q}_{2}^{\dagger 3} . \\
\hat{\mathbb{L}}_{2}^{\dagger}(p) & B_{7}^{\dagger}(p) \hat{\mathbb{L}}_{2}{ }^{1}+\frac{1}{2} B_{7}^{\dagger}(p) \mathbb{L}_{2}{ }^{1}+\frac{\sqrt{2}}{2} d(p) B_{3}^{\dagger}(p) \mathbb{Q}_{3}{ }^{1}-\frac{\sqrt{2}}{2} b(p) B_{3}^{\dagger}(p) \mathbb{Q}_{2}^{\dagger 4},
\end{aligned}
$$




\subsection{Boundary scattering}

We define the two-particle bound state boundary $S$-matrix $R^{B}(p)$ by

$$
B_{J}^{\dagger}(p) \mathbf{B}=R_{J}^{B J^{\prime}}(p) B_{J^{\prime}}^{\dagger}(-p) \mathbf{B} .
$$

We assume, as in the case of the fundamental representation, that the $s u(1 \mid 2)$ generators (2.17) annihilate the vacuum state $|0\rangle_{B}=\mathbf{B}|0\rangle$. Consider now one-particle states $B_{J}^{\dagger}(p)|0\rangle_{B}$. Invariance under $\mathbb{L}_{1}{ }^{1}$ and $\mathbb{R}_{\alpha}^{\beta}$ implies that the boundary $S$-matrix has the structure

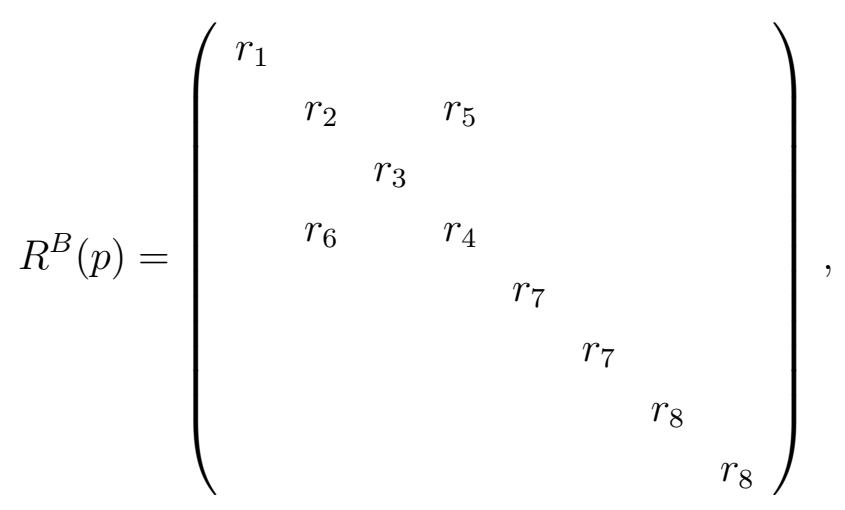

where matrix elements which are zero are left empty. Note that, in contrast with the $l=$ 1 (fundamental representation) case, the $l=2$ (two-particle bound state representation) boundary $S$-matrix is not diagonal.

Invariance under the supersymmetry generators $\mathbb{Q}_{\alpha}{ }^{1}, \mathbb{Q}_{1}^{\dagger \alpha}$ leads to the following set of linear equations,

$$
\begin{aligned}
a(-p) e^{i p / 2} r_{1}-a(p) e^{-i p / 2} r_{7} & =0, \\
a(-p) e^{i p / 2} r_{2}-b(-p) e^{i p / 2} r_{5}-a(p) e^{-i p / 2} r_{8} & =0, \\
b(-p) e^{i p / 2} r_{4}-a(-p) e^{i p / 2} r_{6}-b(p) e^{-i p / 2} r_{8} & =0, \\
a(p) e^{-i p / 2} r_{4}+b(p) e^{-i p / 2} r_{5}-a(-p) e^{i p / 2} r_{7} & =0, \\
b(p) e^{-i p / 2} r_{2}+a(p) e^{-i p / 2} r_{6}-b(-p) e^{i p / 2} r_{7} & =0, \\
b(p) e^{-i p / 2} r_{3}-b(-p) e^{i p / 2} r_{8} & =0,
\end{aligned}
$$




$$
\begin{aligned}
d(p) e^{i p / 2} r_{1}-d(-p) e^{-i p / 2} r_{7} & =0 \\
c(-p) e^{-i p / 2} r_{2}+d(-p) e^{-i p / 2} r_{5}-c(p) e^{i p / 2} r_{7} & =0 \\
d(-p) e^{-i p / 2} r_{4}+c(-p) e^{-i p / 2} r_{6}-d(p) e^{i p / 2} r_{7} & =0 \\
d(p) e^{i p / 2} r_{2}-c(p) e^{i p / 2} r_{6}-d(-p) e^{-i p / 2} r_{8} & =0 \\
c(p) e^{i p / 2} r_{4}-d(p) e^{i p / 2} r_{5}-c(-p) e^{-i p / 2} r_{8} & =0 \\
c(-p) e^{-i p / 2} r_{3}-c(p) e^{i p / 2} r_{8} & =0
\end{aligned}
$$

of which only 6 are independent. Since there are 7 independent matrix elements (any of the eight matrix elements can be set to unity, since we are not concerned with the overall scalar factor), we conclude that the "ordinary" su(1|2) symmetry is not strong enough to determine the boundary $S$-matrix. A similar phenomenon was observed in 9 for the bulk $S$-matrix $S^{B B}$.

We can obtain the needed additional linear equation by assuming that the charge $\tilde{\mathbb{Q}}$ $(2.22)$ is again conserved. Indeed, using (3.9), we find that the action of this charge on the $\mathrm{ZF}$ operators is given by

$$
\begin{aligned}
\tilde{\mathbb{Q}} B_{1}^{\dagger}(p) & =\sqrt{2}\left[-\frac{1}{2} i g u+1-a(p) d(p)\right] B_{2}^{\dagger}(p)-\sqrt{2} a(p) c(p) B_{4}^{\dagger}(p) \\
& +\sqrt{2} B_{2}^{\dagger}(p)\left(\mathbb{L}_{1}{ }^{1}-\mathbb{L}_{2}{ }^{2}\right)+\sqrt{2} c(p) B_{6}^{\dagger}(p) \mathbb{Q}_{3}{ }^{1}-\sqrt{2} c(p) B_{5}^{\dagger}(p) \mathbb{Q}_{4}{ }^{1}+B_{1}^{\dagger}(p) \tilde{\mathbb{Q}}, \\
\tilde{\mathbb{Q}} B_{2}^{\dagger}(p) & =\sqrt{2}\left[-\frac{1}{2} i g u-a(p) d(p)\right] B_{3}^{\dagger}(p)+\sqrt{2} B_{3}^{\dagger}(p)\left(\mathbb{L}_{1}{ }^{1}-\mathbb{L}_{2}{ }^{2}\right) \\
& +c(p) B_{8}^{\dagger}(p) \mathbb{Q}_{3}{ }^{1}-c(p) B_{7}^{\dagger}(p) \mathbb{Q}_{4}{ }^{1}+B_{2}^{\dagger}(p) \tilde{\mathbb{Q}}, \\
\tilde{\mathbb{Q}} B_{3}^{\dagger}(p) & =B_{3}^{\dagger}(p) \tilde{\mathbb{Q}}, \\
\tilde{\mathbb{Q}} B_{4}^{\dagger}(p) & =\sqrt{2} b(p) d(p) B_{3}^{\dagger}(p)-d(p) B_{8}^{\dagger}(p) \mathbb{Q}_{3}{ }^{1}+d(p) B_{7}^{\dagger}(p) \mathbb{Q}_{4}{ }^{1}+B_{4}^{\dagger}(p) \tilde{\mathbb{Q}}, \\
\tilde{\mathbb{Q}} B_{5}^{\dagger}(p) & =-\frac{1}{2} i g u B_{7}^{\dagger}(p)+B_{7}^{\dagger}(p)\left(\mathbb{L}_{1}{ }^{1}-\mathbb{L}_{2}{ }^{2}\right)+\left[d(p) B_{2}^{\dagger}(p)+c(p) B_{4}^{\dagger}(p)\right] \mathbb{Q}_{3}{ }^{1}+B_{5}^{\dagger}(p) \tilde{\mathbb{Q}}, \\
\tilde{\mathbb{Q}} B_{6}^{\dagger}(p) & =-\frac{1}{2} i g u B_{8}^{\dagger}(p)+B_{8}^{\dagger}(p)\left(\mathbb{L}_{1}{ }^{1}-\mathbb{L}_{2}{ }^{2}\right)+\left[d(p) B_{2}^{\dagger}(p)+c(p) B_{4}^{\dagger}(p)\right] \mathbb{Q}_{4}{ }^{1}+B_{6}^{\dagger}(p) \tilde{\mathbb{Q}}, \\
\tilde{\mathbb{Q}} B_{7}^{\dagger}(p) & =\sqrt{2} d(p) B_{3}^{\dagger}(p) \mathbb{Q}_{3}{ }^{1}+B_{7}^{\dagger}(p) \tilde{\mathbb{Q}}, \\
\tilde{\mathbb{Q}} B_{8}^{\dagger}(p) & =\sqrt{2} d(p) B_{3}^{\dagger}(p) \mathbb{Q}_{4}{ }^{1}+B_{8}^{\dagger}(p) \tilde{\mathbb{Q}} .
\end{aligned}
$$

Note that, as in the fundamental case (2.23), all the symmetry generators appearing on the RHS of (3.14) annihilate the vacuum state. 
Consider the action of $\tilde{\mathbb{Q}}$ on the one-particle state $B_{5}^{\dagger}(p)|0\rangle_{B}$. Using (3.14), we obtain

$$
\tilde{\mathbb{Q}} B_{5}^{\dagger}(p)|0\rangle_{B}=-\frac{1}{2} i g u B_{7}^{\dagger}(p)|0\rangle_{B}=-r_{8} \frac{1}{2} i g u B_{7}^{\dagger}(-p)|0\rangle_{B},
$$

where in the first equality we have assumed that $\tilde{\mathbb{Q}}$ and the $s u(1 \mid 2)$ generators (2.17) annihilate the vacuum state; and in the second equality we have "reflected" using (3.10), (3.11). Reversing the order of operations, we obtain

$$
\tilde{\mathbb{Q}} B_{5}^{\dagger}(p)|0\rangle_{B}=r_{7} \tilde{\mathbb{Q}} B_{5}^{\dagger}(-p)|0\rangle_{B}=r_{7} \frac{1}{2} i g u B_{7}^{\dagger}(-p)|0\rangle_{B} .
$$

Comparing (3.15) and (3.16), we arrive at the desired relation 6

$$
r_{7}=-r_{8}
$$

Solving the linear equations (3.12), (3.13), (3.17), we obtain the following result for the boundary $S$-matrix elements

$$
\begin{array}{ll}
r_{1}=1, \quad r_{2}=-\frac{\frac{1}{x^{-}}+x^{-}}{\frac{1}{x^{+}}+x^{-}}, & r_{3}=e^{i p}, \quad r_{4}=\frac{\frac{1}{x^{+}}+x^{+}}{\frac{1}{x^{+}}+x^{-}}, \\
r_{5}=-r_{6}=e^{i p / 2} \frac{x^{-}-x^{+}}{1+x^{-} x^{+}}, & r_{7}=-r_{8}=e^{i p / 2} .
\end{array}
$$

The boundary $S$-matrix (3.11), (3.18) and its Yangian symmetry are our main results. We have verified using Mathematica that this boundary $S$-matrix $R^{B}(p)$ satisfies both boundary Yang-Baxter equations

$$
\begin{aligned}
& S_{12}^{A B}\left(p_{1}, p_{2}\right) R_{1}^{A}\left(p_{1}\right) S_{21}^{B A}\left(p_{2},-p_{1}\right) R_{2}^{B}\left(p_{2}\right) \\
& \quad=R_{2}^{B}\left(p_{2}\right) S_{12}^{A B}\left(p_{1},-p_{2}\right) R_{1}^{A}\left(p_{1}\right) S_{21}^{B A}\left(-p_{2},-p_{1}\right),
\end{aligned}
$$

and

$$
\begin{aligned}
& S_{12}^{B B}\left(p_{1}, p_{2}\right) R_{1}^{B}\left(p_{1}\right) S_{21}^{B B}\left(p_{2},-p_{1}\right) R_{2}^{B}\left(p_{2}\right) \\
& \quad=R_{2}^{B}\left(p_{2}\right) S_{12}^{B B}\left(p_{1},-p_{2}\right) R_{1}^{B}\left(p_{1}\right) S_{21}^{B B}\left(-p_{2},-p_{1}\right),
\end{aligned}
$$

\footnotetext{
${ }^{6}$ By acting with $\tilde{\mathbb{Q}}$ on the one-particle states $B_{1}^{\dagger}(p)|0\rangle_{B}$ and $B_{2}^{\dagger}(p)|0\rangle_{B}$, one can derive in a similar manner the following further relations,

$$
\begin{aligned}
r_{1}\left[\frac{1}{2} i g u-b(-p) c(-p)\right]+r_{2}\left[\frac{1}{2} i g u+b(p) c(p)\right]+r_{6} a(p) c(p) & =0, \\
r_{1} a(-p) c(-p)-r_{4} a(p) c(p)-r_{5}\left[\frac{1}{2} i g u+b(p) c(p)\right] & =0, \\
r_{2}\left[\frac{1}{2} i g u-a(-p) d(-p)\right]+r_{3}\left[\frac{1}{2} i g u+a(p) d(p)\right]+r_{5} b(-p) d(-p) & =0,
\end{aligned}
$$
}

which are also satisfied by the solution (3.18). 
where

$$
S_{21}^{B A}\left(p_{1}, p_{2}\right)=S_{12}^{A B}\left(p_{2}, p_{1}\right)^{-1}
$$

and $R^{A}(p)$ is given by (2.18), (2.19). We note that the boundary unitarity equation

$$
R^{B}(p) R^{B}(-p)=\mathbb{I}
$$

is also satisfied.

\section{Discussion}

We have showed that boundary scattering for the $Y=0$ brane has a residual Yangian symmetry, which we have exploited to determine the boundary $S$-matrix for two-particle bound states. We expect that it should be possible to further exploit this symmetry to determine the boundary $S$-matrices for general $l$-particle bound states. However, this will require developing more powerful techniques, perhaps along the lines of [11]. We also expect that boundary scattering for the $Z=0$ brane [13] has the full Yangian symmetry $Y(\operatorname{su}(2 \mid 2))$, which should determine the corresponding bound state boundary $S$-matrices. Moreover, the boundary $S$-matrices which have been proposed for $D 7$ and $D 5$ branes [20] presumably also have Yangian symmetry. We hope to be able to address these problems in the near future.

The fact that Yangian symmetry has been found in both bulk and boundary scattering suggests that Yangian symmetry may be a generic feature of AdS/CFT worldsheet scattering. It would be interesting to understand if there is any connection with the recently-discovered Yangian symmetry in spacetime scattering [21], [22].

\section{Acknowledgments}

We are grateful to G. Arutyunov, S. Frolov and E. Quinn for valuable correspondence, and for making available to us their code for the bulk $S$-matrix $S^{B B}$. C.A. thanks the University of Miami for hospitality during the course of this work. This work was supported in part by KRF-2007-313-C00150 and WCU grant R32-2008-000-10130-0 (CA), and by the National Science Foundation under Grants PHY-0554821 and PHY-0854366 (RN). 


\section{A Two-particle bound state representation of the $s u(2 \mid 2)$ generators}

We provide here the explicit two-particle bound state representation of the $s u(2 \mid 2)$ generators, which follows from (3.1), (3.2), and which is used for the computations in Sec. 3.

For $\alpha=3,4$,

$$
\begin{aligned}
\mathbb{Q}_{\alpha}{ }^{1}\left|e_{1}\right\rangle & =\sqrt{2} a\left|e_{\alpha+2}\right\rangle, & \mathbb{Q}_{\alpha}{ }^{2}\left|e_{1}\right\rangle & =0 \\
\mathbb{Q}_{\alpha}{ }^{1}\left|e_{2}\right\rangle & =a\left|e_{\alpha+4}\right\rangle, & \mathbb{Q}_{\alpha}{ }^{2}\left|e_{2}\right\rangle & =a\left|e_{\alpha+2}\right\rangle, \\
\mathbb{Q}_{\alpha}{ }^{1}\left|e_{3}\right\rangle & =0, & \mathbb{Q}_{\alpha}{ }^{2}\left|e_{3}\right\rangle & =\sqrt{2} a\left|e_{\alpha+4}\right\rangle, \\
\mathbb{Q}_{\alpha}{ }^{1}\left|e_{4}\right\rangle & =-b\left|e_{\alpha+4}\right\rangle, & \mathbb{Q}_{\alpha}{ }^{2}\left|e_{4}\right\rangle & =b\left|e_{\alpha+2}\right\rangle, \\
\mathbb{Q}_{\alpha}{ }^{1}\left|e_{5}\right\rangle & =-\delta_{\alpha}^{4}\left(b\left|e_{2}\right\rangle+a\left|e_{4}\right\rangle\right), & \mathbb{Q}_{\alpha}{ }^{2}\left|e_{5}\right\rangle & =\delta_{\alpha}^{4} \sqrt{2} b\left|e_{1}\right\rangle, \\
\mathbb{Q}_{\alpha}{ }^{1}\left|e_{6}\right\rangle & =\delta_{\alpha}^{3}\left(b\left|e_{2}\right\rangle+a\left|e_{4}\right\rangle\right), & \mathbb{Q}_{\alpha}{ }^{2}\left|e_{6}\right\rangle & =-\delta_{\alpha}^{3} \sqrt{2} b\left|e_{1}\right\rangle, \\
\mathbb{Q}_{\alpha}{ }^{1}\left|e_{7}\right\rangle & =-\delta_{\alpha}^{4} \sqrt{2} b\left|e_{3}\right\rangle, & \mathbb{Q}_{\alpha}{ }^{2}\left|e_{7}\right\rangle & =\delta_{\alpha}^{4}\left(b\left|e_{2}\right\rangle-a\left|e_{4}\right\rangle\right) \\
\mathbb{Q}_{\alpha}{ }^{1}\left|e_{8}\right\rangle & =\delta_{\alpha}^{3} \sqrt{2} b\left|e_{3}\right\rangle, & \mathbb{Q}_{\alpha}{ }^{2}\left|e_{8}\right\rangle & =\delta_{\alpha}^{3}\left(-b\left|e_{2}\right\rangle+a\left|e_{4}\right\rangle\right),
\end{aligned}
$$

and

$$
\begin{array}{rlrl}
\mathbb{Q}_{1}^{\dagger \alpha}\left|e_{1}\right\rangle & =0, & \mathbb{Q}_{2}^{\dagger \alpha}\left|e_{1}\right\rangle & =-\sqrt{2} c \epsilon^{\alpha \beta}\left|e_{\beta+2}\right\rangle, \\
\mathbb{Q}_{1}^{\dagger \alpha}\left|e_{2}\right\rangle & =c \epsilon^{\alpha \beta}\left|e_{\beta+2}\right\rangle, & \mathbb{Q}_{2}^{\dagger \alpha}\left|e_{2}\right\rangle=-c \epsilon^{\alpha \beta}\left|e_{\beta+4}\right\rangle, \\
\mathbb{Q}_{1}^{\dagger \alpha}\left|e_{3}\right\rangle=\sqrt{2} c \epsilon^{\alpha \beta}\left|e_{\beta+4}\right\rangle, & \mathbb{Q}_{2}^{\dagger \alpha}\left|e_{3}\right\rangle=0, \\
\mathbb{Q}_{1}^{\dagger \alpha}\left|e_{4}\right\rangle=d \epsilon^{\alpha \beta}\left|e_{\beta+2}\right\rangle, & \mathbb{Q}_{2}^{\dagger \alpha}\left|e_{4}\right\rangle=d \epsilon^{\alpha \beta}\left|e_{\beta+4}\right\rangle, \\
\mathbb{Q}_{1}^{\dagger \alpha}\left|e_{5}\right\rangle=\delta_{3}^{\alpha} \sqrt{2} d\left|e_{1}\right\rangle, & \mathbb{Q}_{2}^{\dagger \alpha}\left|e_{5}\right\rangle=\delta_{3}^{\alpha}\left(d\left|e_{2}\right\rangle+c\left|e_{4}\right\rangle\right), \\
\mathbb{Q}_{1}^{\dagger \alpha}\left|e_{6}\right\rangle=\delta_{4}^{\alpha} \sqrt{2} d\left|e_{1}\right\rangle, & \mathbb{Q}_{2}^{\dagger \alpha}\left|e_{6}\right\rangle=\delta_{4}^{\alpha}\left(d\left|e_{2}\right\rangle+c\left|e_{4}\right\rangle\right), \\
\mathbb{Q}_{1}^{\dagger \alpha}\left|e_{7}\right\rangle=\delta_{3}^{\alpha}\left(d\left|e_{2}\right\rangle-c\left|e_{4}\right\rangle\right), & \mathbb{Q}_{2}^{\dagger \alpha}\left|e_{7}\right\rangle=\delta_{3}^{\alpha} \sqrt{2} d\left|e_{3}\right\rangle, \\
\mathbb{Q}_{1}^{\dagger \alpha}\left|e_{8}\right\rangle=\delta_{4}^{\alpha}\left(d\left|e_{2}\right\rangle-c\left|e_{4}\right\rangle\right), & \mathbb{Q}_{2}^{\dagger \alpha}\left|e_{8}\right\rangle=\delta_{4}^{\alpha} \sqrt{2} d\left|e_{3}\right\rangle .
\end{array}
$$

Moreover, 


$$
\begin{aligned}
\mathbb{L}_{1}^{2}\left|e_{1}\right\rangle & =0, & \mathbb{L}_{2}{ }^{1}\left|e_{1}\right\rangle & =\sqrt{2}\left|e_{2}\right\rangle, \\
\mathbb{L}_{1}{ }^{2}\left|e_{2}\right\rangle & =\sqrt{2}\left|e_{1}\right\rangle, & \mathbb{L}_{2}{ }^{1}\left|e_{2}\right\rangle & =\sqrt{2}\left|e_{3}\right\rangle, \\
\mathbb{L}_{1}{ }^{2}\left|e_{3}\right\rangle & =\sqrt{2}\left|e_{2}\right\rangle, & \mathbb{L}_{2}{ }^{1}\left|e_{3}\right\rangle & =0, \\
\mathbb{L}_{1}{ }^{2}\left|e_{4}\right\rangle & =0, & \mathbb{L}_{2}{ }^{1}\left|e_{4}\right\rangle & =0, \\
\mathbb{L}_{1}{ }^{2}\left|e_{5}\right\rangle & =0, & \mathbb{L}_{2}{ }^{1}\left|e_{5}\right\rangle & =\left|e_{7}\right\rangle, \\
\mathbb{L}_{1}{ }^{2}\left|e_{6}\right\rangle & =0, & \mathbb{L}_{2}{ }^{1}\left|e_{6}\right\rangle & =\left|e_{8}\right\rangle, \\
\mathbb{L}_{1}{ }^{2}\left|e_{7}\right\rangle & =\left|e_{5}\right\rangle, & \mathbb{L}_{2}{ }^{1}\left|e_{7}\right\rangle & =0, \\
\mathbb{L}_{1}{ }^{2}\left|e_{8}\right\rangle & =\left|e_{6}\right\rangle, & \mathbb{L}_{2}{ }^{1}\left|e_{8}\right\rangle & =0,
\end{aligned}
$$

and $\mathbb{R}_{3}{ }^{4}\left|e_{J}\right\rangle=0=\mathbb{R}_{4}{ }^{3}\left|e_{J}\right\rangle$ except for the following:

$$
\begin{array}{ll}
\mathbb{R}_{3}^{4}\left|e_{6}\right\rangle=\left|e_{5}\right\rangle, & \mathbb{R}_{3}^{4}\left|e_{8}\right\rangle=\left|e_{7}\right\rangle, \\
\mathbb{R}_{4}{ }^{3}\left|e_{5}\right\rangle=\left|e_{6}\right\rangle, & \mathbb{R}_{4}^{3}\left|e_{7}\right\rangle=\left|e_{8}\right\rangle .
\end{array}
$$

Finally, $\mathbb{L}_{1}{ }^{1}, \mathbb{L}_{2}{ }^{2}, \mathbb{R}_{3}{ }^{3}, \mathbb{R}_{4}^{4}$ are represented by diagonal matrices, whose eigenvalues are given in the following table:

\begin{tabular}{|r|r|r|r|r|}
\hline & $\mathbb{L}_{1}^{1}$ & $\mathbb{L}_{2}^{2}$ & $\mathbb{R}_{3}^{3}$ & $\mathbb{R}_{4}{ }^{4}$ \\
\hline$\left|e_{1}\right\rangle$ & 1 & -1 & 0 & 0 \\
$\left|e_{2}\right\rangle$ & 0 & 0 & 0 & 0 \\
$\left|e_{3}\right\rangle$ & -1 & 1 & 0 & 0 \\
$\left|e_{4}\right\rangle$ & 0 & 0 & 0 & 0 \\
$\left|e_{5}\right\rangle$ & $\frac{1}{2}$ & $-\frac{1}{2}$ & $\frac{1}{2}$ & $-\frac{1}{2}$ \\
$\left|e_{6}\right\rangle$ & $\frac{1}{2}$ & $-\frac{1}{2}$ & $-\frac{1}{2}$ & $\frac{1}{2}$ \\
$\left|e_{7}\right\rangle$ & $-\frac{1}{2}$ & $\frac{1}{2}$ & $\frac{1}{2}$ & $-\frac{1}{2}$ \\
$\left|e_{8}\right\rangle$ & $-\frac{1}{2}$ & $\frac{1}{2}$ & $-\frac{1}{2}$ & $\frac{1}{2}$ \\
\hline
\end{tabular}

\section{References}

[1] M. Staudacher, "The factorized S-matrix of CFT/AdS," JHEP 0505, 054 (2005) arXiv:hep-th/0412188.

[2] N. Beisert, "The $s u(2 \mid 2)$ dynamic S-matrix," Adv. Theor. Math. Phys. 12, 945 (2008) arXiv:hep-th/0511082; 
N. Beisert, "The Analytic Bethe Ansatz for a Chain with Centrally Extended su(2|2) Symmetry," J. Stat. Mech. 0701, P017 (2007) arXiv:nlin/0610017.

[3] G. Arutyunov, S. Frolov and M. Zamaklar, "The Zamolodchikov-Faddeev algebra for $A d S_{5} \times S^{5}$ superstring," JHEP 0704, 002 (2007) arXiv:hep-th/0612229].

[4] N. Beisert, "The S-Matrix of AdS/CFT and Yangian Symmetry," PoS(SOLVAY) 002 (2006) arXiv:0704.0400].

[5] L. Dolan, C. R. Nappi and E. Witten, "A relation between approaches to integrability in superconformal Yang-Mills theory," JHEP 0310, 017 (2003) arXiv:hep-th/0308089]; L. Dolan, C. R. Nappi and E. Witten, "Yangian symmetry in $D=4$ superconformal Yang-Mills theory," arXiv:hep-th/0401243.

[6] A. Agarwal and S. G. Rajeev, "Yangian symmetries of matrix models and spin chains: The dilatation operator of $\mathcal{N}=4$ SYM," Int. J. Mod. Phys. A 20, 5453 (2005) arXiv:hep-th/0409180.

[7] N. Berkovits, "Quantum consistency of the superstring in $A d S_{5} \times S^{5}$ background," JHEP 0503, 041 (2005) arXiv:hep-th/0411170.

[8] B. I. Zwiebel, "Yangian symmetry at two-loops for the $s u(2 \mid 1)$ sector of $\mathcal{N}=4$ SYM," J. Phys. A 40, 1141 (2007) arXiv:hep-th/0610283.

[9] G. Arutyunov and S. Frolov, "The S-matrix of String Bound States," Nucl. Phys. B 804, 90 (2008) arXiv:0803.4323 [hep-th]].

[10] M. de Leeuw, "Bound States, Yangian Symmetry and Classical r-matrix for the $A d S_{5} \times$ $S^{5}$ Superstring," JHEP 0806, 085 (2008) [arXiv:0804.1047 [hep-th]].

[11] G. Arutyunov, M. de Leeuw and A. Torrielli, "The Bound State $S$-Matrix for $A d S_{5} \times S^{5}$ Superstring," Nucl. Phys. B 819, 319 (2009) arXiv:0902.0183 [hep-th]].

[12] P.P. Kulish, N.Yu. Reshetikhin and E.K. Sklyanin, "Yang-Baxter equation and representation theory. I," Lett. Math. Phys. 5, 393 (1981);

P.P. Kulish and E.K. Sklyanin, "Quantum spectral transform method, recent developments," Lecture Notes in Physics 151, 61 (Springer, 1982).

[13] D.M. Hofman and J.M. Maldacena, "Reflecting magnons," JHEP 0711, 063 (2007) arXiv:0708.2272 [hep-th]].

[14] C. Ahn and R.I. Nepomechie, "The Zamolodchikov-Faddeev algebra for open strings attached to giant gravitons," JHEP 0805, 059 (2008) [arXiv:0804.4036 [hep-th]]. 
[15] A.B. Zamolodchikov and Al.B. Zamolodchikov, "Factorized $S$ matrices in twodimensions as the exact solutions of certain relativistic quantum field models," Ann. Phys. 120, 253 (1979).

[16] L.D. Faddeev, "Quantum completely integral models of field theory," Sov. Sci. Rev. C1, 107 (1980).

[17] S. Ghoshal and A.B. Zamolodchikov, "Boundary S-Matrix and Boundary State in TwoDimensional Integrable Quantum Field Theory," Int. J. Mod. Phys. A9, 3841 (1994) arXiv:hep-th/9306002.

[18] G. W. Delius, N. J. MacKay and B. J. Short, "Boundary remnant of Yangian symmetry and the structure of rational reflection matrices," Phys. Lett. B 522, 335 (2001) [Erratum-ibid. B 524, 401 (2002)] arXiv:hep-th/0109115.

[19] A. Doikou, "On reflection algebras and boundary Yangians," J. Math. Phys. 46, 053504 (2005) arXiv:hep-th/0403277.

[20] D. H. Correa and C. A. S. Young, "Reflecting magnons from D7 and D5 branes," J. Phys. A 41, 455401 (2008) [arXiv:0808.0452 [hep-th]].

[21] J. M. Drummond, J. M. Henn and J. Plefka, "Yangian symmetry of scattering amplitudes in $\mathcal{N}=4$ super Yang-Mills theory," JHEP 0905, 046 (2009) arXiv:0902.2987 [hep-th]].

[22] N. Beisert, J. Henn, T. McLoughlin and J. Plefka, "One-Loop Superconformal and Yangian Symmetries of Scattering Amplitudes in $\mathcal{N}=4$ Super Yang-Mills," arXiv:1002.1733 [hep-th]]. 